\title{
Violation of cell lineage restriction compartments in the chick hindbrain
}

\author{
Eric Birgbauer and Scott E. Fraser \\ Division of Biology, 139-74, Beckman Institute, California Institute of Technology, Pasadena, California 91125, USA
}

\section{SUMMARY}

Previous cell lineage studies indicate that the repeated neuromeres of the chick hindbrain, the rhombomeres, are cell lineage restriction compartments. We have extended these results and tested if the restrictions are absolute. Two different cell marking techniques were used to label cells shortly after rhombomeres form (stage 9+ to 13) so that the resultant clones could be followed up to stage 25 . Either small groups of cells were labelled with the lipophilic dye DiI or single cells were injected intracellularly with fluorescent dextran. The majority of the descendants labelled by either technique were restricted to within a single rhombomere. However, in a small but reproducible proportion of the cases (greater than $5 \%$ ), the clones expanded across a rhombomere boundary. Neither the stage of injection, the stage of analysis, the dorsoventral position, nor the rhombomere identity correlated with the boundary crossing. Judging from the morphology of the cells, both neurons and non-neuronal cells were able to expand over a boundary. These results demonstrate that the rhombomere boundaries represent cell lineage restriction barriers which are not impenetrable in normal development.

Key words: rhombomere, hindbrain, lineage, compartments, boundaries, LRD, DiI

\section{INTRODUCTION}

The possibility that the nervous system is constructed from a segmented set of repeated units has become an issue of intense interest in recent years (see Lumsden, 1990; Keynes and Lumsden, 1990; Fraser, 1993). A series of undulations of the neural tube, termed neuromeres (Orr, 1887), have been documented for over a century (for review, see Vaage, 1969; Bergquist, 1952). Because the neuromeres represent a transient organization during the development of the central nervous system (Vaage, 1969), many questions about their importance have been raised. For example, are the neuromeres an underlying organizational pattern of the central nervous system, or are they merely a consequence of external morphogenetic pressures? The criteria for segmentation in the brain, and elsewhere, have been under active discussion (see Lumsden, 1990; Keynes and Lumsden, 1990; Shankland, 1991); criteria have ranged from cellular or anatomical patterns associated with the neuromeres, cell lineage or cell proliferation compartments, to patterns of gene expression coincident with the neuromeres. Recent work makes it readily apparent that not all neuromeres are alike. For example, in the avian spinal cord, the profound segmentation of the motor and sensory nerves do not appear to represent an intrinsic segmentation of the nervous system. There is not a segmental pattern of neurogenesis in the chick spinal cord (Lim et al., 1991), nor does it demonstrate intrinsic cell lineage compartments (Stern et al., 1991). Instead, it appears that the segmental organization arises through interactions with the adjacent somites (Lim et al., 1991).

A body of work is now emerging which suggests that the neuromeres of the hindbrain, termed rhombomeres, reflect an intrinsic segmentation of the neural tube. DiI backfilling of the cranial motor nerves shows a segmental organization (Lumsden and Keynes, 1989). For example in chick, the fifth cranial (trigeminal) nerve traces to neuronal cell bodies in rhombomeres 2 and 3. The seventh cranial (facial) nerve is derived primarily from cell bodies in rhombomeres 4 and 5. The ninth cranial (glossopharyngeal) nerve comes from the projections of neurons in rhombomeres 6 and 7. In addition, neurogenesis shows a pattern paralleling the rhombomere organization. Developing neurons, recognized by neurofilament antibody staining, are found first in rhombomeres 2, 4, and 6, followed later by rhombomeres 3, 5, and 7 (Lumsden and Keynes, 1989). Furthermore, some cell surface antigens are expressed in a segmental manner coincident with the rhombomeres, suggesting intrinsic differences in the neuroepithelium (Kuratani, 1991; Layer and Alber, 1990; Trevarrow et al., 1990; Kuratani and Eichele, 1993).

Searches for possible molecular correlates to the rhombomeres have centered on the pattern of expression of several putative regulatory genes, including genes of the Antennapedia class of homeobox-containing (Hox) genes (see Hunt et al., 1991a,b) and the zinc-finger gene Krox-20 (Wilkinson et al., 1989a,b; Nieto et al., 1991; Hunt et al., 1991c). For example, in both chick and mouse, Hox-Bl is specifically expressed in rhombomere 4 shortly after rhombomere formation (Murphy et al., 1989; Sundin and Eichele, 1990), and Krox-20 is expressed in presumptive rhombomeres 3 and 5 even before their appearance (Wilkinson et al., 1989a; Nieto et al., 1991). The expression pattern of other members of the Hox cluster appears to coincide with rhombomere boundaries; in addition, differing levels of expression result in distinct patterns of gene expression in each of the different rhombomeres, raising the possibility of a molecular code (Wilkinson et al., 1989b; Wilkinson and Krumlauf, 1990; Hunt et al., 1991a,c). 
The cellular basis of the rhombomere organization has been explored using intracellular injections of large fluorescent dyes to trace cell lineage in the hindbrain. Cells labelled before rhombomere boundary formation yielded some clones that cross rhombomere boundaries; however, cells labelled after rhombomere formation yielded clones that were restricted to only one rhombomere, even though these clones sometimes expanded to fill much of the rostrocaudal extent of the rhombomere (Fraser et al., 1990). The absence of cells that crossed the boundaries has been used to define the rhombomeres as compartments of cell lineage restriction. We have extended these results by documenting the position and timing of the injections with respect to the already established rhombomere boundaries. Labeling small groups of cells with DiI, or single cells intracellularly with fluorescent dextran, revealed that although the vast majority of clones are restricted to a single rhombomere, a small proportion are seen to expand over a rhombomere boundary. We have examined these 'violator' clones in more detail, examining potential correlates with stage, position, and morphology.

\section{MATERIALS AND METHODS}

\section{Chick embryos}

Fertile White Leghorn chicken eggs (or sometimes Rhode Island Red) were obtained from Chino Valley Ranchers (Chino, CA). Eggs were incubated at $38^{\circ} \mathrm{C}$ on their sides in a rocking incubator for $40-46$ hours to stage 10 to 13 (Hamburger and Hamilton, 1951). Embryos were lowered away from the top of the egg by removing about $1.5 \mathrm{ml}$ of albumen using a $3 \mathrm{ml}$ syringe with an $18 \mathrm{G}$ needle inserted into the round end of the egg. A 1-2 cm diameter hole was cut into the egg shell above the embryo. A 1:10 dilution of Pelikan Fount India Ink in Howard's Ringer $\left(0.12 \mathrm{M} \mathrm{NaCl}, 1.5 \mathrm{mM} \mathrm{CaCl}_{2}, 5 \mathrm{mM} \mathrm{KCl}\right)$ was injected sub-blastodermally to visualize the embryos. After staging (Hamburger and Hamilton, 1951), the vitelline membrane was gently torn open above the hindbrain with a tungsten needle. The initial injections were made as described below into either ventral, lateral, or dorsal-lateral neural tube; no injections were made in the dorsalmedial neural tube to minimize the complication of labelling neural crest cells. The injections were visualized briefly under epifluorescence and compared with the bright-field image to determine their location. In the case of DiI injections, the positions of the labelled cells were documented by recording the fluorescence and bright-field images with a Newvicon video camera onto $\frac{3}{4}$ inch video tape. Finally, $1-3$ drops of a $5 \%$ solution of penicillin/streptomycin $(25,000 \mathrm{U} / \mathrm{ml}$ stock, Whittaker Bioproducts, Walkersville, MD) in Howard's Ringer (or albumen) were added to the egg. The egg was then sealed with tape and allowed to incubate in a humidified, non-rocking incubator at $38^{\circ} \mathrm{C}$ for a further $1-2$ days (to stage $15-25$ ).

\section{Dyes and injections}

Lysinated rhodamine dextran (LRD) and 1,1'-dioctadecyl-3,3,3',3'tetramethylindocarbocyanine, perchlorate (DiI) were obtained from Molecular Probes (Eugene, OR). A $3 \times 10^{3} M_{\mathrm{r}}$ LRD (catalog no. D3308 ) was used in some injections, while a $10 \times 10^{3} M_{\mathrm{r}} \mathrm{LRD}$ (catalog no. D-1817) was used in others.

Electrodes were pulled from Al-Si glass capillaries (with filament) using a Sutter P-80/PC Micropipette Puller. Electrodes of 10-30 M $\Omega$ resistance were used for LRD injections, and 5-10 $\mathrm{M} \Omega$ resistance for DiI injections.

LRD injections of single cells were performed as described previously (Bronner-Fraser and Fraser, 1989; Fraser et al., 1990). Briefly, the tip was back-loaded with $100 \mathrm{mg} / \mathrm{ml} \mathrm{LRD} \mathrm{in} \mathrm{water,} \mathrm{and} \mathrm{the}$ electrode was then back-filled with $1.2 \mathrm{M} \mathrm{LiCl}$. For microinjection, the tip was lowered into the neural tube and rung briefly with the negative capacitance control until a membrane potential was recorded. LRD was injected iontophoretically with $4 \mathrm{nA}$ current for 10 seconds with the $3 \times 10^{3} \mathrm{M}_{\mathrm{r}} \mathrm{LRD}$ or for 30 seconds with the $10 \times 10^{3} \mathrm{M}_{\mathrm{r}} \mathrm{LRD}$. Most embryos had a single cell labelled per embryo. However, a few embryos received LRD injections into 2 or 3 spatially separated single cells. If there was more than one injection into a single embryo, the injections were separated by two rhombomeres or were on opposite sides of the midline.

DiI injections were performed by back-loading electrodes with $0.5 \%$ DiI in ethanol. The electrodes were placed in a holder with a silver wire that was immersed into the DiI solution. After positioning the electrodes into the neural tube, DiI was injected iontophoretically with $90 \mathrm{nA}$ current for 2-10 seconds using a box powered by a 9 volt battery through a $100 \mathrm{M} \Omega$ resistor.

\section{Analysis}

After incubation, the embryos were washed in Howard's Ringer, the trunk was removed, and the heads were fixed for $16-20$ hours at $4{ }^{\circ} \mathrm{C}$ with $4 \%$ paraformaldehyde in $0.1 \mathrm{M}$ phosphate buffer, $\mathrm{pH} 7.4$. For DiI injected embryos, the fix solution also contained $0.25 \%$ glutaraldehyde. After fixation and washing in PBS, the hindbrain was carefully dissected out. The roofplate was cut and the hindbrain laid flat on a slide with the ventricular surface up (Lumsden and Keynes, 1989).

Clones were scored under epifluorescence microscopy and rhombomere boundaries were visualized by bright-field illumination using a Zeiss axiophot microscope. Alignment of the clones with the rhombomere boundaries was assessed by imaging with a SIT camera (Hamamatsu) and superimposing the epifluorescence and bright-field images. Many of the embryos were also examined on a Biorad MRC600 laser confocal mounted on a Zeiss axiovert microscope. The confocal microscope was used to collect a z-series at $5 \mu \mathrm{m}$ intervals, which was compressed computationally into a single plane and overlaid onto the bright-field image.

The rhombomere boundary appears in bright-field illumination as a phase dense undulation of the neural tube of about 2-3 cells in width. Only those clones in which there were clearly labelled cells on both sides of this somewhat broad boundary line were deemed to have crossed the boundary. Those clones that had labelled cells in this boundary region, but not on the opposite side, were deemed not to have crossed the boundary. If the label crossing a boundary appeared to be only in long, thin processes (presumably axons) rather than cell bodies, it was not scored as crossing the boundary. If there was a single spot of label crossing the boundary, which could possibly be a growth cone, but no axon was visible, this clone was tabulated separately as a probable boundary crosser.

\section{RESULTS}

\section{Dil lineage analysis}

To investigate the restrictions to cell mixing at a rhombomere boundary in the chick hindbrain, we followed small groups of cells labelled with the fluorescent lipophilic dye DiI. DiI was injected iontophoretically into a single rhombomere at stage 9+ to 13 in chick (Hamburger and Hamilton, 1951), after rhombomere boundaries have appeared. The labelled cells were visualized briefly after injection, recorded using a Newvicon video camera, and their location noted. An example of such an injection is shown in Fig. 1A. The injections were small, usually labelling 1-12 cells. If the initially labelled group of DiI-marked cells was seen to cross into a second rhombomere, that embryo was discarded. 
After the injected embryos were incubated to stages 15-23, they were fixed, and their excised hindbrains were flatmounted to examine the localization of the DiI-labelled cells. Fig. 1 shows such an analysis on three embryos. In a majority of the embryos, the DiI-labelled cells were restricted to within one rhombomere (Fig. 1B). A minority of the embryos showed a pattern of DiI-labelled cells crossing a rhombomere boundary. For example, Fig. 1C depicts the same embryo as in Fig. 1A after it has developed to stage 21. The DiI was initially localized within rostral rhombomere 3 (Fig 1A); however, by stage 21 , some of the labelled cells had crossed over the $\mathrm{r} 2 / \mathrm{r} 3$ boundary into caudal r2 (Fig 1C). Similarly, Fig. 1D shows a case in which the initial label was in mid to rostral r3; by stage 23 , the majority of the labelled cells are in $\mathrm{r} 3$, but some labelled cells are found in r2. Of 112 embryos analyzed, 97 (87\%) showed restriction of the DiI label to cells within a single rhombomere. In 15 embryos (13\%), some DiI-labelled cells were seen to cross over a boundary into a second rhombomere (summarized in Table 1). Of these 15 embryos, there were nine cases in which label crossing the boundary was small and might be a growth cone. To clearly segregate these cases, we refer to them as 'probable'. However, 6 cases $(5 \%)$ had cell bodies that unquestionably crossed a rhombomere boundary.

A histogram of the clone behavior versus the stage of
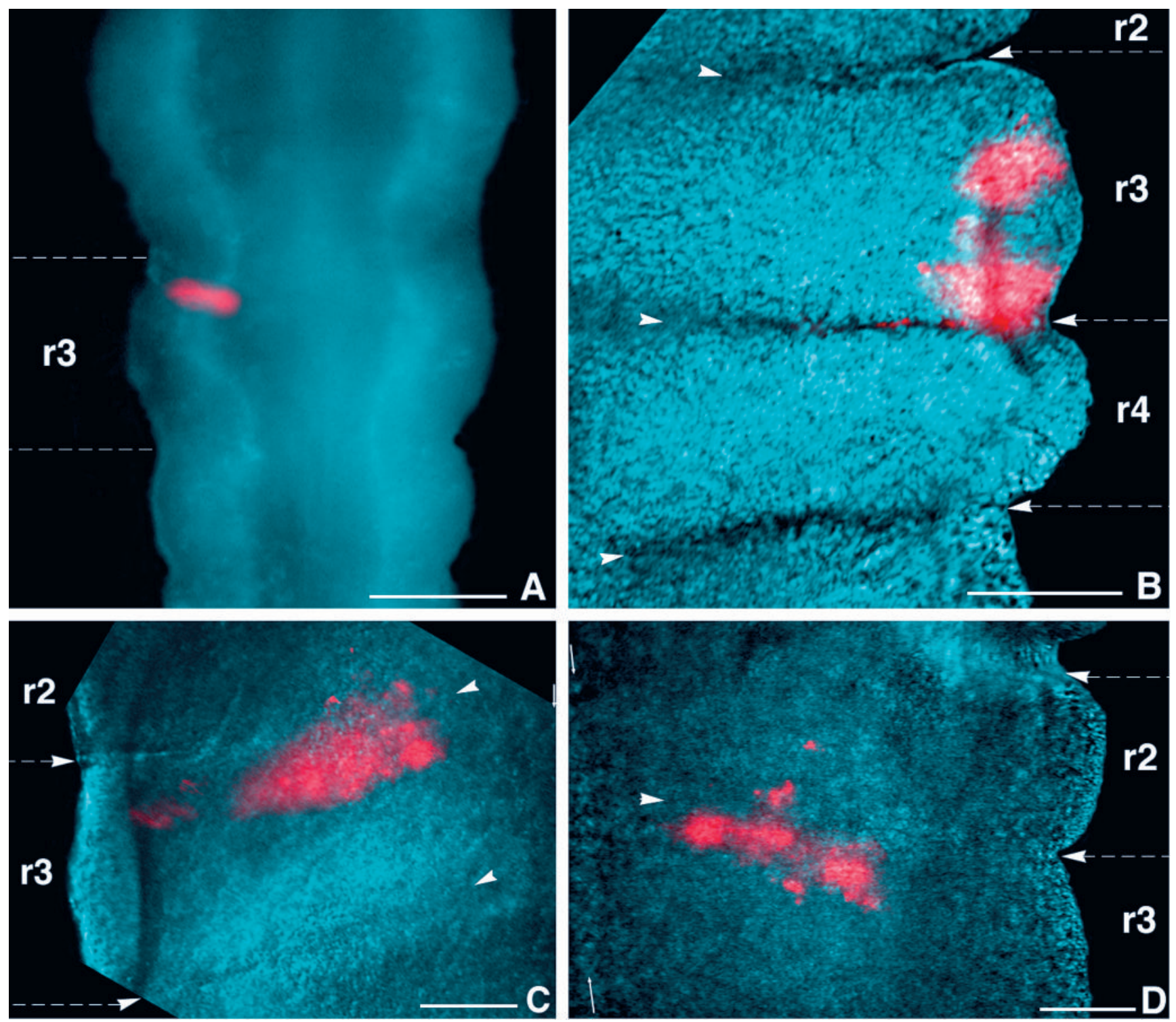

Fig. 1. DiI labelling of small groups of cells in the chick hindbrain. (A) DiI labelling of a small group of cells immediately after injection into rostral rhombomere 3 (r3) of a stage 10 chick embryo. The fluorescence image is slightly blurred because of the light scattering by overlying tissue and the differing depths of the labelled cells. (B-D) Examples of DiI-labelled embryos analyzed later showing DiI fluorescence (red) superimposed over the bright-field image (blue). Excised hindbrains were flat-mounted by deflecting the dorsal margins to the right and left. (B) Stage 17 chick embryo showing label entirely within rhombomere 3, including an axon in the r3/r4 boundary. (C) Same embryo as in A above, allowed to develop to stage 21, showing DiI label in rhombomere 3 and rhombomere 2. (D) Another embryo, stage 23, showing DiI label in rhombomere 2 and rhombomere 3 . Arrowheads point to rhombomere boundaries (dark bands in bright-field image). Small arrows indicate the midline of that hindbrain. Bars, $100 \mu \mathrm{m}$. 
Table 1. DiI-labelled cell groups that cross boundaries

\begin{tabular}{|c|c|c|c|c|}
\hline Boundary crossed & Position & Stage at fixation & Stage at injection & Injection site \\
\hline $\mathrm{r} 2 / \mathrm{r} 3 \dagger$ & Dorsal & $17+$ & 12 & r3 (rostral) \\
\hline $\mathrm{r} 2 / \mathrm{r} 3$ & Dorsal & $19+$ & 10 & r3 (mid) \\
\hline $\mathrm{r} 2 / \mathrm{r} 3$ & Dorsal & $17-$ & $10+$ & r3 (mid) \\
\hline $\mathrm{r} 2 / \mathrm{r} 3 \dagger$ & Dorsal to mid & $21+$ & $12-$ & r3 (rostral) \\
\hline $\mathrm{r} 2 / \mathrm{r} 3$ & Dorsal to mid & 21 & $11-$ & r3 (rostral) \\
\hline $\mathrm{r} 2 / \mathrm{r} 3 \dagger$ & Dorsal to mid & 21 & $11-$ & r3 (rostral) \\
\hline $\mathrm{r} 2 / \mathrm{r} 3$ & Dorsal to mid & $19+$ & $10-$ & r3 (mid) \\
\hline $\mathrm{r} 2 / \mathrm{r} 3$ & Mid & 23 & $12-$ & r3 (mid-rostral) \\
\hline $\mathrm{r} 2 / \mathrm{r} 3 \dagger$ & Mid & 17 & $10+$ & r3 (mid-rostral) \\
\hline $\mathrm{r} 2 / \mathrm{r} 3 \dagger$ & Mid to ventral & 17 & $13-$ & r3 (rostral) \\
\hline $\mathrm{r} 2 / \mathrm{r} 3$ & Ventral & 21 & 10 & r3 (rostral) \\
\hline $\mathrm{r} 2 / \mathrm{r} 3 \dagger$ & Ventral & $17-$ & $11+$ & r3 (mid) \\
\hline $\mathrm{r} 3 / \mathrm{r} 4 \dagger$ & Mid & $21-$ & 11 & r3 (mid) \\
\hline $\mathrm{r} 3 / \mathrm{r} 4 \dagger$ & Ventral & 20 & $9+$ & r3 (mid) \\
\hline $\mathrm{r} 3 / \mathrm{r} 4 \dagger$ & Ventral & 19 & $10-$ & r3 (mid) \\
\hline
\end{tabular}

Embryos in which the DiI labeled cells cross a rhombomere boundary. The dorsal/ventral position where the labeled cells cross the boundary is given in the second column. The location of the injected cell within the neural tube was determined immediately after injection (injection site). All embryos were staged

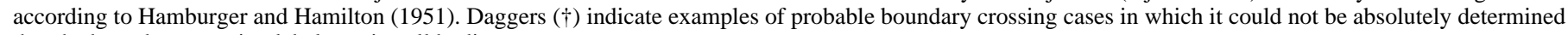
that the boundary-crossing label was in cell bodies.

fixation was constructed to determine if there was a correlation of boundary crossing with embryonic stage (Fig. 2). A comparison of the number of embryos at each stage in which the DiI clearly crossed a boundary (clear bar) and those that probably crossed a boundary (light stipple) with those in which the DiI label was restricted to a single rhombomere (dark stipple) suggests that there is no critical period for boundary violation.

To determine if there was a dorsoventral bias to rhombomere boundary crossing, the dorsoventral position of the DiIlabelled cells was catalogued. Three regions of the neural tube were delineated: the dorsal third, the ventral third, and the middle third, and the position of the DiI-labelled cells were categorized accordingly. If the labelled cells spread to more than one region, the results from that embryo were counted in both categories. 55 embryos contained DiI label in the dorsal region, 4 of which (7\%) clearly crossed a rhombomere boundary. 40 contained DiI-labelled cells in the ventral third, with $1(2 \%)$ clearly crossing a boundary. 64 embryos contained DiI label in the mid region, with $3(5 \%)$ clearly crossing a boundary. If the probable boundary crossing cases are added, 7 (13\%) cross a rhombomere boundary in the dorsal region, $5(12 \%)$ cross in the ventral region, and $8(12 \%)$ cross in the mid region. The similarity of these fractions suggests there is no strong dorsoventral bias in the probability that cells cross a rhombomere boundary.

\section{LRD lineage analysis}

To ensure that the DiI results were not due to the transfer of the vital dye, we performed similar analyses using the hydrophilic fluorescent dye LRD (lysinated rhodamine dextran). LRD was injected iontophoretically into single cells, allowing us to follow clones, since LRD does not partition between cells, either directly or through gap junctions. LRD injections were made into the chick neural tube at stages 1013 (Hamburger and Hamilton, 1951), shortly after rhombomere boundaries appear. Embryos were briefly examined under epifluorescence illumination immediately after injection to determine whether we had labelled only a single cell. Unlike previous lineage analyses, if more than 1 cell was labelled by a single injection, the embryo was not discarded; instead, this was noted and the embryo was kept for later analysis. We examined 89 clones derived from single neural tube cells marked at stages $10-13$ and surviving to stages $18-25$. We also examined 19 cases where there was more than one cell labelled initially but where all the labelled cells were clearly localized within one rhombomere.

Similar to the DiI results, a majority of the clones were restricted to a single rhombomere. Fig. 3 shows two examples of clones that lie completely within one rhombomere. For example, in Fig. 3A, a clone of cells with processes (see inset)

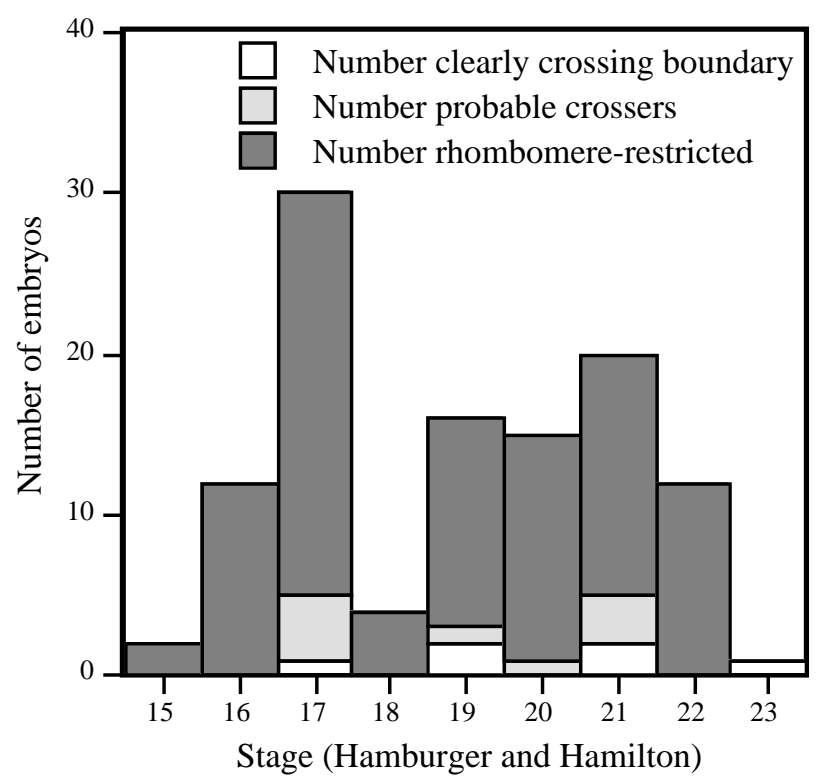

Fig. 2. Histogram of embryonic stage of boundary crossing versus rhombomere restriction in DiI-labelled embryos. At each stage, the number of embryos containing DiI-labelled cells clearly crossing a rhombomere boundary (clear bar) and probably crossing a rhombomere boundary (light stipple) are compared with the number of embryos in which the DiI-labelled cells were completely restricted to one rhombomere (dark stipple). 
abuts but does not cross the rostral border of rhombomere 3 . Fig. 3B shows a clone of cells which abuts the r3/r4 boundary, but does not cross it. In addition, a minority of clones were not restricted to a single rhombomere (Fig. 4). Fig. 4A shows one of the few embryos in which two separate injections were made. The upper clone of cells in Fig. 4A clearly straddles the $\mathrm{r} 3 / \mathrm{r} 4$ boundary, with half of the clone in each rhombomere. In the lower clone, most of the label is in r5, but there is a labelled spot in r6 (large arrow). There were several clones in which a single spot of label crossed a rhombomere boundary. Since it was difficult to be certain that this spot was a cell and not a growth cone in which the axon staining was not visible, all analyses were performed both including and excluding these probable boundary-crossing cases. Fig. 4B shows another example of a crossing clone, with the majority of the clone in $\mathrm{r} 3$, but some labelled cells in $\mathrm{r} 2$. Fig. 4C shows a clone that lies mostly within r6, but a group of cells clearly is in r5.

Of the 89 clones derived from neural tube cells marked at stages $10-13$ and surviving to stages $18-25,74(83 \%)$ were restricted to a single rhombomere; 8 clones $(9 \%)$ clearly were not restricted to a single rhombomere, and 7 (8\%) were probable for boundary crossing. As with the DiI-labelled cells, if the label that crossed the boundary was clearly present in only axons or growth cones, the clone was not scored as crossing the boundary. Each of the non-restricted clones is listed in Table 2. A similar fraction of rhombomere boundary crossing was observed in those 19 cases in which more than 1 cell was injected initially: the labelled descendants of $16(84 \%)$ were contained within a single rhombomere; in $1(5 \%)$ of these cases, the labelled descendants clearly crossed a rhombomere boundary, and in $2(11 \%)$ cases, it was probable that the label crossed a rhombomere boundary. These 3 cases are denoted with asterisks (*) in Table 2 . As these non-clonal cases were similar to the clonal cases in frequency of boundary crossing, phenotype, and apparent clone size, the data have been combined. In total, of the 108 cases of labelled cells in embryos surviving to stage $18-25,90(83 \%)$ lay within one rhombomere, while $9(8 \%)$ clearly crossed a rhombomere boundary and another $9(8 \%)$ were probable for crossing a rhombomere boundary.

\section{Properties of boundary crossing clones}

Various properties of the clones were examined to determine if there was a correlation with boundary crossing. A histogram of the number of clones that crossed a boundary (both clear and probable cases) shows they were found throughout the range of stages examined, in roughly the same proportion as those that were restricted to a single rhombomere (Fig. 5). Similarly, there was no correlation between the stage of injection and boundary crossing.

Clonal extent was examined to determine if clones that crossed rhombomere boundaries had expanded to fill a greater fraction of the rhombomere. Clonal extent was measured as the rostrocaudal extent of the clone relative to the size of the rhombomere. The 89 clones that were restricted to a single rhombomere had a rostrocaudal extent ranging from $10 \%$ to $90 \%$ of a rhombomere, with an average of $35 \pm 15$ (mean \pm s.d.) percent of a rhombomere. The 8 clear boundary crossing clones had an average rostrocaudal extent of $55 \pm 30$ percent of a rhombomere (range: $20 \%$ to $110 \%$ of a rhombomere length). With such a large variation in extent, the boundary-crossing clones are not significantly different from the rhombomere-restricted clones. The results from the probable boundary crossing cases and from LRD injection in which more than one cell was labelled were not significantly different. There were no cases in which a group of LRD-labelled cells crossed two rhombomere boundaries.

Correlation with axial level was examined in two ways: at the level of the different rhombomeres within the hindbrain and by the position within a rhombomere. Unlike the DiI injections, which were concentrated into rhombomere 3 , we injected LRD into cells in a range of rhombomeres. A summary of the

Table 2. LRD clones that cross boundaries

\begin{tabular}{|c|c|c|c|c|}
\hline Boundary crossed & Cell morphology & Stage at fixation & Stage at injection & Injection site \\
\hline $\mathrm{r} 1 / \mathrm{r} 2$ & Processes & $21-$ & $11-$ & $\mathrm{r} 2$ \\
\hline $\mathrm{r} 1 / \mathrm{r} 2 *$ & Processes & 19 & $11-$ & r2 (rostral) \\
\hline $\mathrm{r} 2 / \mathrm{r} 3$ & No processes & 21 & 10 & r3 (rostral) \\
\hline $\mathrm{r} 2 / \mathrm{r} 3 \dagger$ & No processes & $20-21$ & 11 & r3 (rostral) \\
\hline $\mathrm{r} 2 / \mathrm{r} 3$ & Processes & $21-$ & $10+$ & r3 (rostral) \\
\hline $\mathrm{r} 2 / \mathrm{r} 3$ & Processes & $19+$ & $11+$ & r3 (rostral) \\
\hline $\mathrm{r} 2 / \mathrm{r} 3 \dagger$ & Both & 25 & $13+$ & r3 (rostral) \\
\hline $\mathrm{r} 2 / \mathrm{r} 3^{*} \dagger$ & Processes & $19+$ & 11 & r3 (rostral) \\
\hline $\mathrm{r} 2 / \mathrm{r} 3 \dagger$ & No processes & $21-$ & 12 & r3 (mid) \\
\hline $\mathrm{r} 3 / \mathrm{r} 4$ & No processes & 21 & $12-$ & r3 (mid) \\
\hline $\mathrm{r} 3 / \mathrm{r} 4$ & Processes & $20+$ & $12-$ & r3 (mid) \\
\hline $\mathrm{r} 3 / \mathrm{r} 4 \dagger$ & Processes & 18 & 10 & r3 (caudal) \\
\hline $\mathrm{r} 3 / \mathrm{r} 4 *+$ & Both & $21-$ & $10+$ & r4 (rostral) \\
\hline $\mathrm{r} 3 / \mathrm{r} 4 \dagger$ & Processes & $20+$ & 12 & r4 (mid) \\
\hline $\mathrm{r} 4 / \mathrm{r} 5 \dagger$ & Processes & $20-$ & 10 & $\mathrm{r} 4$ \\
\hline $\mathrm{r} 5 / \mathrm{r} 6 \dagger$ & Processes & $20+$ & $12-$ & r5 (mid) \\
\hline r5/r6 & Both & $23+$ & 11 & r6 (mid) \\
\hline $\mathrm{r} 6 / \mathrm{r} 7$ & Processes & $21-$ & $10+$ & r6 (mid) \\
\hline
\end{tabular}

All the clones derived from LRD single cell injections that crossed a rhombomere boundary at the time of fixation. Asterisks (*) indicate cases that were derived from an initial injection of 2 or more cells within one rhombomere. Daggers $(\dagger)$ indicate examples of probable boundary crossing cases in which it could not be absolutely determined that the boundary-crossing label was in cell bodies. The cellular morphology of a clone was classified based on whether the cells

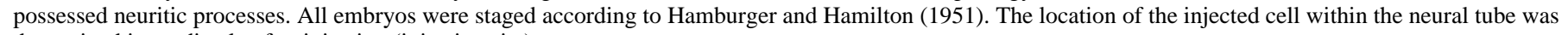
determined immediately after injection (injection site). 
location of both the rhombomere restricted and boundary crossing clones (Fig. 6) shows that both rhombomere restricted clones and boundary crossing clones were found at each axial level. No particular boundary is a more major obstacle than another. We tabulated the number of restricted clones and boundary crossing clones (both clearly crossing and probable) from injections at each rhombomere level (Table 3). The percentage of clones that crossed a rhombomere boundary was roughly equivalent for each rhombomere. In addition, clones were seen to cross rhombomere boundaries both from rostral and from caudal with no indication of a restricted direction. Surprisingly, we found that boundary-crossing clones could come from cells injected at any axial level within a rhombomere. Boundary crossing clones came not only from injections at the rostral or caudal edges, but also from the middle of a rhombomere (see Table 2).

To determine if there was a correlation of cell type with boundary crossing, we analyzed the morphology of the LRD-filled cells within the clones. Based on the LRD staining, clones were classified as containing cells with processes, presumably neurons, or as containing cells with no discernible processes. Those clones that appeared to have process-bearing cells and cells without processes were classified as 'both'. Because of the density of label, it was often hard to determine if clones that had cells bearing processes also had cells that did not have processes. Thus it is possible that the class labelled 'both' could be underrepresented, or even overrepresented. However, this potential pitfall should occur equally among the restricted and boundary crossing clones. There were examples of rhombomere-restricted and boundary crossing clones in each class of cell morphology (Table 4). The distribution of clones among morphology classes was similar for the rhombomere-restricted and boundary crossing clones.

\section{DISCUSSION}

We examined cell lineage restrictions in the chick hindbrain by following cells labelled by two methods: DiI labelling of small groups of cells and LRD injection of single cells. Both methods show that most clones are restricted by the rhombomere boundaries in the hindbrain. However, a small number of clones $(5-17 \%)$ are not restricted by the rhombomere boundaries. Some of these cases might result from misidentification of growth cones crossing the boundary, but others were definitely cells that had crossed a rhombomere boundary.

Analyses to date have failed to define any specific subclass of cells that are responsible for these boundary crossers. There was no strong correlation with the stage of labelling or the stage of analysis. The slight tendency for more DiI-labelled boundary crossers to be found at later stages may well be due
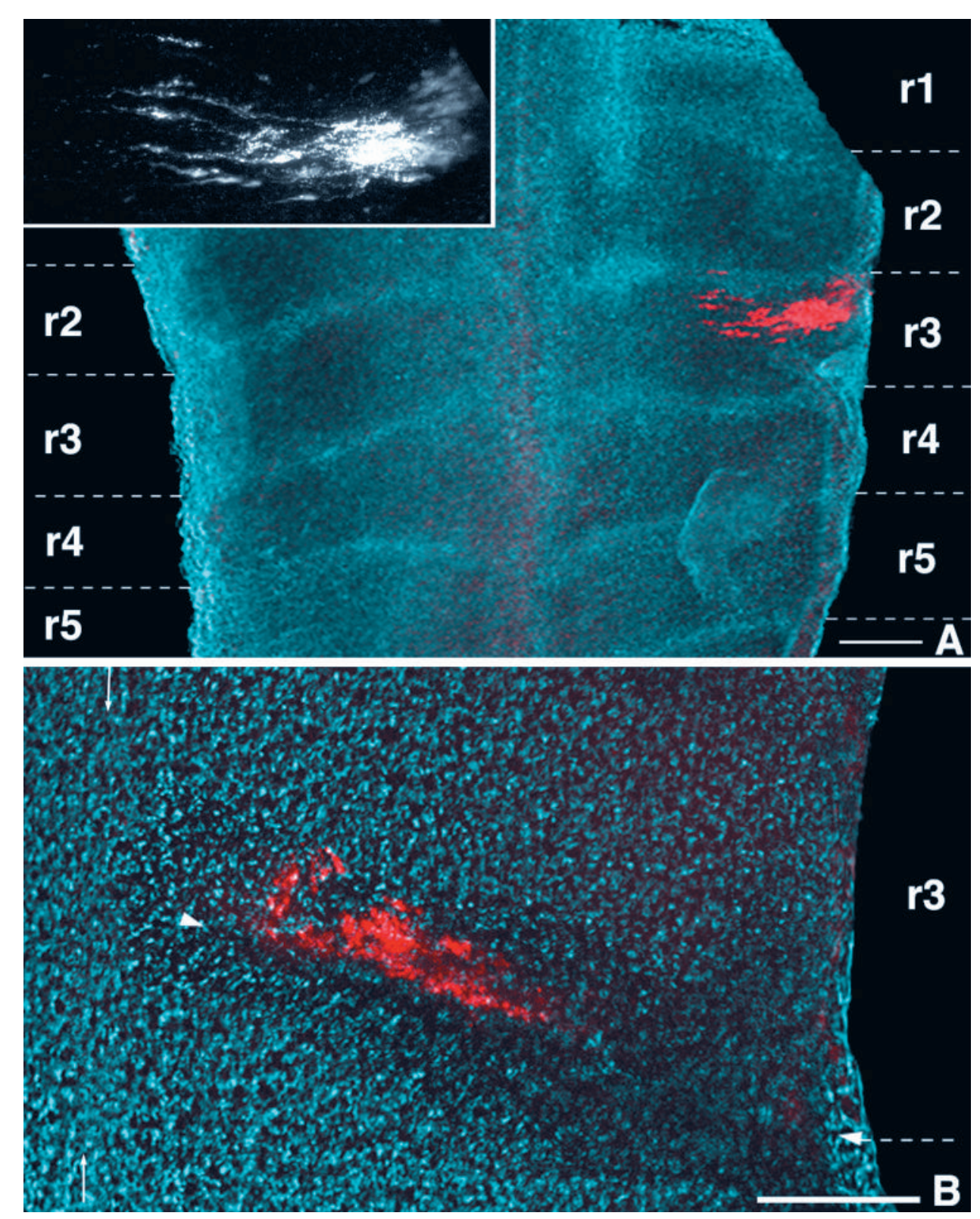

Fig. 3. Flat-mount hindbrains showing LRD-labelled clones of cells that lie within one rhombomere. A confocal z-series of the LRD fluorescence (red) is shown superimposed over the bright-field image (blue). (A) Flat-mount hindbrain from a stage 20 chick showing a clone of LRDlabelled cells that lies entirely within rhombomere 3 (r3). The inset, a two-fold magnification of the fluorescent image, shows the axonal processes more clearly. (B) A clone of LRD-labelled cells in caudal rhombomere 3 in a stage 24 chick. This clone abuts the r3/r4 boundary (arrowheads), but does not cross it. Small arrows indicate the ventral midline in B. Scale bars, $100 \mu \mathrm{m}$. 
to the clones expanding more by later stages. No dorsoventral pattern to boundary crossing was found. Each rhombomere boundary showed crossing clones; thus, boundary crossing does not seem to be a property of only certain rhombomeres. One may have expected to see a pattern emerge where a group of rhombomeres would form a lineage restriction compartment. For instance, the two rhombomere repeat of the cranial nerves (Lumsden and Keynes, 1989) might suggest a two rhombomere compartment. However, no larger pattern was seen as every boundary had cases of clones that crossed over it. Also, within a rhombomere, boundary crossing did not seem to be dependent on the injection site. There were restricted clones and boundary crossing clones from injections into the rostral part of the rhombomere, the middle part of the rhombomere, and the caudal part of the rhombomere. No correlation was found with boundary crossing and whether the clones contained cells with dye-filled processes. Based on the length and position of these processes, these cells were most likely neurons. We did not determine which neuronal types cross rhombomere boundaries because of the small number of boundary-crossing clones and the difficulty of tracing the LRD-filled axons. Therefore, it remains to be determined whether all neuronal types can cross rhombomere boundaries. There were also clones with cells in which no dyefilled processes were discernible. These cells without processes could be glial cells, progenitor cells, undifferentiated neurons, or even differentiated neurons in which the processes were too faintly labelled to see; as a result, clones classified as 'no processes' or 'both' could be neurons. However, even with the limitations of this analysis, it is clear that boundary crossing clones were not restricted to one morphological class. Of course, with neuronal clones, we cannot distinguish whether differentiated neurons crossed rhombomere boundaries or whether a progenitor cell or an undifferentiated neuron crossed a boundary and then differentiated later.

In a previous analysis, Fraser et al. (1990) did not find any clones that crossed a rhombomere boundary when a cell was labelled after rhombomere boundary formation. Of the 30 cells injected after rhombomere boundaries had formed, all the resultant clones were restricted to a single rhombomere at stages 18-19. They
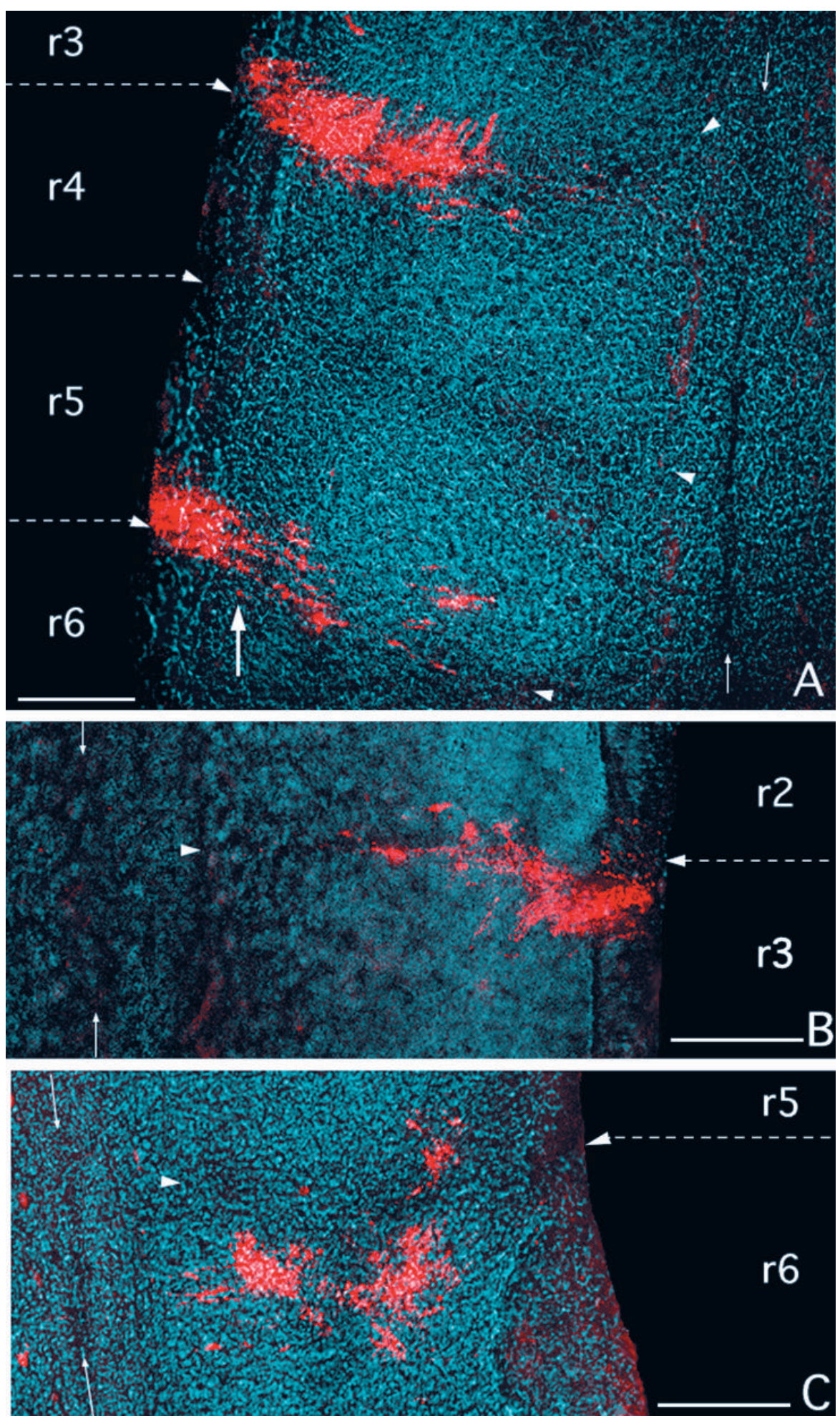

Fig. 4. Examples of LRD clones that cross a rhombomere boundary. A confocal z-series of the fluorescent clone (red) is shown superimposed on the bright-field image (blue). (A) Two LRD-labelled clones in flat-mount hindbrain of a stage 20 chick. The more rostral clone crosses the $\mathrm{r} 3 / \mathrm{r} 4$ boundary. The more caudal clone is probable in that it lies within rhombomere 5 except for one spot of label in r6 (denoted by large arrow). (B) A clone from a stage 21 chick that crosses the $\mathrm{r} 2 / \mathrm{r} 3$ boundary. (C) A clone from a stage 23 chick that has many cells in rhombomere 6 , but several cells within $\mathrm{r} 5$ as well. Arrowheads point to rhombomere boundaries, which appear dark in bright-field illumination. Small arrows denote the midline of each hindbrain flat-mount. Scale bars, $100 \mu \mathrm{m}$. 


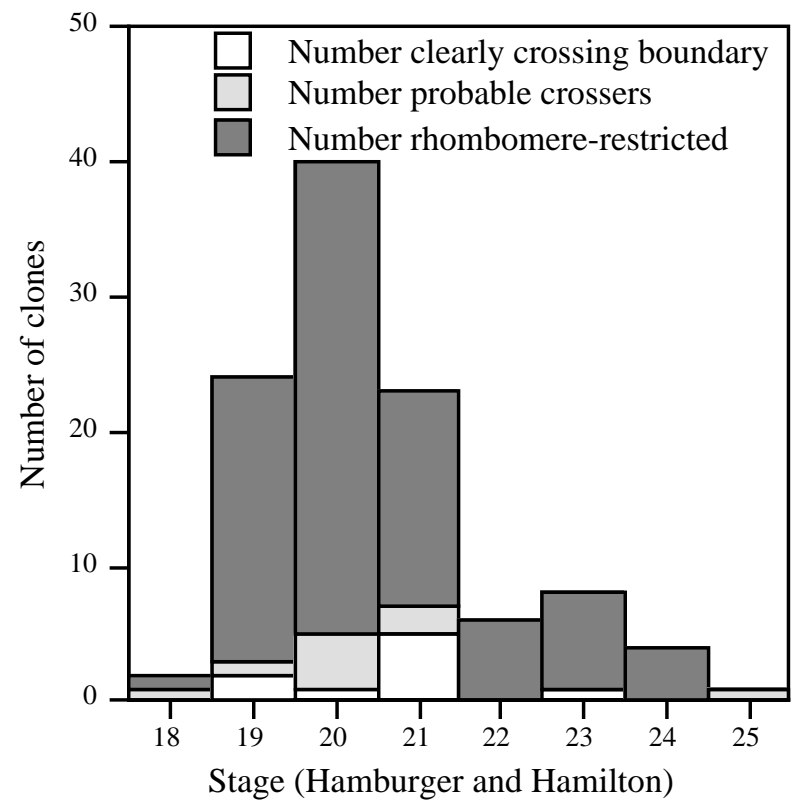

Fig. 5. Bar graph showing the number of LRD clones examined after fixation at each stage. The clear bar shows the number of clones at that stage that clearly crossed a rhombomere boundary, and the light stipple shows the number of probable boundary crossing clones. The dark stipple shows the number of clones at that stage that lay entirely within one rhombomere.

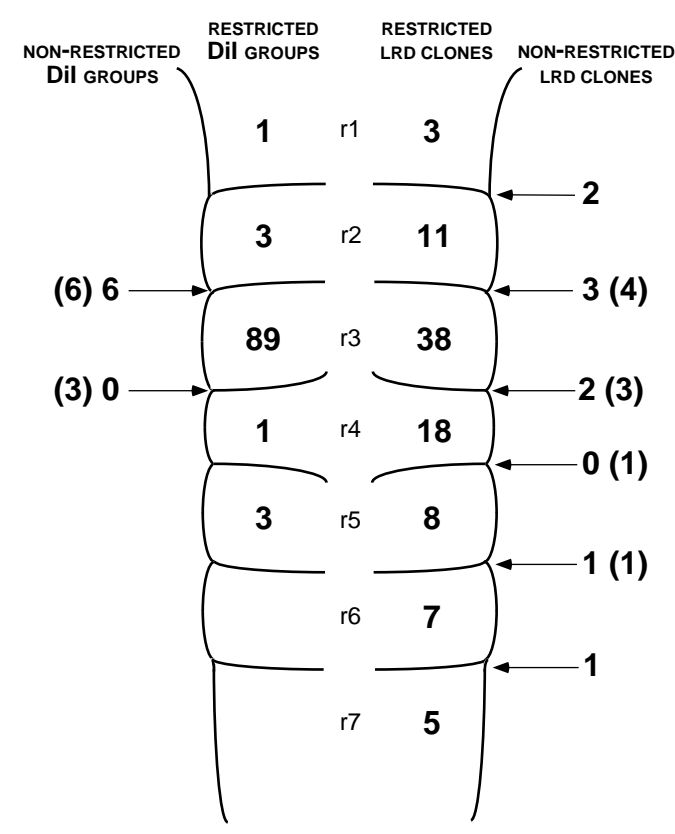

Fig. 6. Schematic diagram of the chick hindbrain showing the location of the clones analyzed. The left half shows the position of DiI-labelled groups of cells and the right half shows the position of LRD-labelled clones. Within the hindbrain is the number of clones at each axial level that lay completely within that rhombomere. Beside the hindbrain is the number of clones that clearly crossed that rhombomere boundary (arrows), with the number of probable boundary-crossing clones shown in parentheses. No clones were found that crossed more than 1 boundary.
Table 3. Position of LRD clones

\begin{tabular}{lccccc}
\hline $\begin{array}{l}\text { Rhombomere } \\
\text { injected }\end{array}$ & $n$ & $\begin{array}{c}\text { Restricted } \\
n(\%)\end{array}$ & $\begin{array}{c}\text { Crossing } \\
\text { rostral } \\
n(\%)\end{array}$ & $\begin{array}{c}\text { Crossing } \\
\text { caudal } \\
n(\%)\end{array}$ & $\begin{array}{c}\text { Total } \\
\text { crossing } \\
n(\%)\end{array}$ \\
\hline r1 & 3 & $3(100)$ & 0 & 0 & 0 \\
r2 & 13 & $11(85)$ & $2(15)$ & 0 & $2(15)$ \\
r3 & 48 & $38(79)$ & $7(15)$ & $3(6)$ & $10(21)$ \\
r4 & 21 & $18(86)$ & $2(10)$ & $1(5)$ & $3(14)$ \\
r5 & 9 & $8(89)$ & 0 & $1(11)$ & $1(11)$ \\
r6 & 9 & $7(78)$ & $1(11)$ & $1(11)$ & $2(22)$ \\
r7 & 5 & $5(100)$ & 0 & 0 & 0
\end{tabular}

The number of injections into each rhombomere are shown along with the number of clones derived from these injections that were restricted to that rhombomere, and the number that crossed over a boundary. For this table, we have included both clear and probable cases of rhombomere boundary crossing. Excluding the probable cases shows fewer clones but no significant differences. The total crossed over are further divided into those that crossed rostrally or caudally from the injected rhombomere. The percentage restricted or crossing compared with the total number of injections into that rhombomere are shown in parentheses.

therefore concluded that the rhombomere boundaries were absolute lineage restriction boundaries. We have labelled 108 cells with LRD after boundary formation (stages 10-13) and obtained 9 clones $(8 \%)$ that clearly cross a rhombomere boundary at stages 18-25, and an additional $9(8 \%)$ that probably cross a boundary, indicating that rhombomere boundaries are not absolute lineage restriction boundaries. How do we reconcile these results? First, most of the clones reported here were examined at a later stage than those of Fraser et al. (1990); however, we find some clones crossing boundaries even at stages 18-19 (Fig. 5). Confirming this, in our DiI labelling experiments, we saw evidence of boundary crossing as early as stage 17 (Fig. 2). Thus, failure of lineage restriction at rhombomere boundaries does not seem to be strongly stage dependent. Another possibility is that the small number of injections (30) of Fraser et al. (1990) just did not catch the minority result. A vast majority $(80-95 \%)$ of our clones are clearly restricted to a single rhombomere. With only $8 \%$ of the clones clearly crossing the rhombomere boundaries, it could be possible to miss boundary crossing clones with only 30 injections. In the present study, it is possible that some of the fluorescent structures identified may be growth cones rather than cell bodies, so we were careful to count these as probables. However, even excluding these probable cases, there were several cases in which large numbers of clearly identified cell bodies had obviously violated the boundary (Figs 1C, 4C).

Although our experiments indicate that the rhombomere boundaries are not absolute lineage restriction boundaries, they still must be significant lineage restriction barriers. The number

Table 4. Morphology of LRD clones

\begin{tabular}{lccc}
\hline Types of clones & Processes $(\%)$ & No processes $(\%)$ & Both $(\%)$ \\
\hline Within 1 rhombomere & $70(78)$ & $13(14)$ & $7(8)$ \\
Clearly crosses boundary & $6(67)$ & $2(22)$ & $1(11)$ \\
Probably crosses boundary & $5(56)$ & $2(22)$ & $2(22)$ \\
Total & $81(75)$ & $17(16)$ & $10(9)$
\end{tabular}

The number of clones containing cells with processes, cells without processes, or both. The percentage of clones in each morphology class is given in parentheses. 
of clones restricted to one rhombomere is greater than would be expected if clones could freely expand over rhombomere boundaries. Given that the average rostrocaudal extent of all clones is $40 \%$ of the extent of a rhombomere, one would predict that a clone should end up expanding over a rhombomere boundary in about $40 \%$ of the cases. Because only 8 $17 \%$ of the clones expand over a rhombomere boundary (depending on whether probable cases are counted), it appears that the boundaries act as significant, albeit imperfect, lineage restriction barriers. Although such violations are unexpected based upon early discussions of compartments in insects (see Lawrence and Morata, 1976), recent work shows clear cases of violations of segmental boundaries (Vincent and O'Farrell, 1992) and perhaps even parasegmental boundaries (S. E. F. and J.-P. Vincent, unpublished findings) in Drosophila embryos.

The eventual fate of the cells that crossed a rhombomere boundary has not yet been determined. There are several possibilities. For example, they could be recognized as being in the wrong compartment and then eliminated by a mechanism of cell death. This cannot be an immediate response since some of the boundary crossing clones had many cells in the 'wrong' rhombomere, some even with processes. Instead, the boundary crossing cells may be induced by neighboring cells to adopt a new fate in accord with their new location. This would not be surprising considering the changes of gene expression for 'identity' genes, such as Krox-20 and the Hox genes, as the rhombomeres are set up (Wilkinson et al., 1989a,b; Murphy et al., 1989; Sundin and Eichele, 1990; Murphy and Hill, 1991; Nieto et al., 1991, 1992; Hunt et al., 1991c). For example, antibodies against the Hox-B1 product show expressing cells to be mixed with non-expressing cells, followed later by the expression band including all r4 cells (Sundin and Eichele, 1990, 1992). However, the stages at which we have seen clones crossing rhombomere boundaries are after most of the 'identity' genes show uniform and intense labelling of cells within a single rhombomere. Thus, the boundary crossing we observe would seem to require a rapid and complete switch in gene expression; otherwise, a small number of cells must have been missed by previous in situ and antibody analyses. Finally, it is possible that the cells that cross into a neighboring rhombomere do not change their fate, but rather maintain the fate of the rhombomere they came from. This would result in some intermixing between adjacent rhombomeres, at least at the border regions; the sharp pattern of expression of putative 'identity' genes at these later stages makes this scenario seem unlikely.

The specific pattern of Hox gene expression, morphological segmentation of the neural tube, and lineage restriction compartments all are set up only to disappear a few stages later. What could be the possible relevance for the transient creation of this highly ordered pattern in the development of the hindbrain? One possible role is that this pattern may be utilized as a scaffold for proper neuronal pathfinding. Indeed, in a HoxAl knock-out mouse in which the rhombomere pattern is disrupted, the projections of motor nerves are aberrant (Carpenter et al., 1993). Another possible function of the segmentation could be to establish building blocks that cooperate to organize the nuclei of the brainstem (Glover and Petursdottir, 1991). Another possibility is that the rhombomeres compartmentalize the hindbrain to provide spatially restricted domains for localized cell interactions. Of course, these possi- bilities are not mutually exclusive; an understanding of their relative roles, if any, in the segmentation of the hindbrain must await further experimental analyses.

We would like to thank Amy Bih-Jwo Lin for help with the dextran injections, and Marianne Bronner-Fraser, Jack Sechrist, Bill Trevarrow, and George Serbedzija for critical reading of the manuscript. E. B. is a Fellow of The Jane Coffin Childs Memorial Fund for Medical Research. This investigation has been aided by a grant from The Jane Coffin Childs Memorial Fund for Medical Research and the NIH (HD-26864).

\section{REFERENCES}

Bergquist, H. (1952). Studies on the cerebral tube in vertebrates. The neuromeres. Acta Zool. (Stockh.) 33, 117-187.

Bronner-Fraser, M. and Fraser, S. (1989). Developmental potential of avian trunk neural crest cells in situ. Neuron 3, 755-766.

Carpenter, E. M., Goddard, J. M., Chisaka, O., Manley, N. R. and Capecchi, M. R. (1993). Loss of Hox-Al (Hox-1.6) function in the reorganization of the murine hindbrain. Development 118, 1063-1075.

Fraser, S., Keynes, R. and Lumsden, A. (1990). Segmentation in the chick embryo hindbrain is defined by cell lineage restrictions. Nature 344, 431435.

Fraser, S. E. (1993). Segmentation moves to the fore. Curr. Biol. 3, 787-789.

Glover, J. C. and Petursdottir, G. (1991). Regional specificity of developing reticulospinal, vestibulospinal, and vestibulo-ocular projections in the chicken embryo. J. Neurobiol. 22, 353-376.

Hamburger, V. and Hamilton, H. L. (1951). A series of normal stages in the development of the chick embryo. J. Exp. Zool. 111, 457-502.

Hunt, P., Gulisano, M., Cook, M., Sham, M. H., Faiella, A., Wilkinson, D., Boncinelli, E. and Krumlauf, R. (1991a). A distinct Hox code for the branchial region of the vertebrate head. Nature 353, 861-864.

Hunt, P., Whiting, J., Muchamore, I., Marshall, H. and Krumlauf, R. (1991b). Homeobox genes and models for patterning the hindbrain and branchial arches. Development Supplement 1, 187-196.

Hunt, P., Whiting, J., Nonchev, S., Sham, M. H., Marshall, H., Graham, A., Cook, M., Allemann, R., Rigby, P. W., Gulisano, M., Faiella, A., Boncinelli, E. and Krumlauf, R. (1991c). The branchial Hox code and its implications for gene regulation, patterning of the nervous system and head evolution. Development Supplement 2, 63-77.

Keynes, R. and Lumsden, A. (1990). Segmentation and the origin of regional diversity in the vertebrate central nervous system. Neuron 4, 1-9.

Kuratani, S. C. (1991). Alternate expression of the HNK-1 epitope in rhombomeres of the chick embryo. Dev. Biol. 144, 215-219.

Kuratani, S. C. and Eichele, G. (1993). Rhombomere transplantation repatterns the segmental organization of cranial nerves and reveals cellautonomous expression of a homeodomain protein. Development 117, 105117.

Lawrence, P. A. and Morata, G. (1976). The compartment hypothesis. In Insect Development (ed. P. A. Lawrence), pp. 132-148. Oxford, England: Blackwell.

Layer, P. G. and Alber, R. (1990). Patterning of chick brain vesicles as revealed by peanut agglutinin and cholinesterases. Development 109, 613624.

Lim, T. M., Jaques, K. F., Stern, C. D. and Keynes, R. J. (1991). An evaluation of myelomeres and segmentation of the chick embryo spinal cord. Development 113, 227-238.

Lumsden, A. (1990). The cellular basis of segmentation in the developing hindbrain. Trends Neurosci. 13, 329-335.

Lumsden, A. and Keynes, R. (1989). Segmental patterns of neuronal development in the chick hindbrain. Nature 337, 424-428.

Murphy, P., Davidson, D. R. and Hill, R. E. (1989). Segment-specific expression of a homoeobox-containing gene in the mouse hindbrain. Nature 341, 156-159.

Murphy, P. and Hill, R. E. (1991). Expression of the mouse labial-like homeobox-containing genes, Hox 2.9 and Hox 1.6, during segmentation of the hindbrain. Development 111, 61-74.

Nieto, M. A., Bradley, L. C., Hunt, P., Das Gupta, R., Krumlauf, R. and Wilkinson, D. G. (1992). Molecular mechanisms of pattern formation in the vertebrate hindbrain. Ciba Found. Symp. 165, 92-107. 
Nieto, M. A., Bradley, L. C. and Wilkinson, D. G. (1991). Conserved segmental expression of Krox-20 in the vertebrate hindbrain and its relationship to lineage restriction. Development Supplement 2, 59-62.

Orr, H. A. (1887). Contribution to the embryology of the lizard. J. Morphol. 1, 311-372.

Shankland, M. (1991). Leech segmentation: cell lineage and the formation of complex body patterns. Dev. Biol. 144, 221-231.

Stern, C. D., Jaques, K. F., Lim, T. M., Fraser, S. E. and Keynes, R. J. (1991). Segmental lineage restrictions in the chick embryo spinal cord depend on the adjacent somites. Development 113, 239-244.

Sundin, O. H. and Eichele, G. (1990). A homeo domain protein reveals the metameric nature of the developing chick hindbrain. Genes Dev. 4, 1267 1276.

Sundin, O. and Eichele, G. (1992). An early marker of axial pattern in the chick embryo and its respecification by retinoic acid. Development 114, 841852.

Trevarrow, B., Marks, D. L. and Kimmel, C. B. (1990). Organization of hindbrain segments in the zebrafish embryo. Neuron 4, 669-679.
Vaage, S. (1969). The segmentation of the primitive neural tube in chick embryos. Adv. Anat. Embryol. Cell Biol. 41, 1-87.

Vincent, J.-P. and O'Farrell, P. H. (1992). The state of engrailed expression is not clonally transmitted during early Drosophila development. Cell 68, 923-931.

Wilkinson, D. G., Bhatt, S., Chavrier, P., Bravo, R. and Charnay, P. (1989a). Segment-specific expression of a zinc-finger gene in the developing nervous system of the mouse. Nature 337, 461-464.

Wilkinson, D. G., Bhatt, S., Cook, M., Boncinelli, E. and Krumlauf, R. (1989b). Segmental expression of Hox-2 homeobox-containing genes in the developing mouse hindbrain. Nature 341, 405-409.

Wilkinson, D. G. and Krumlauf, R. (1990). Molecular approaches to the segmentation of the hindbrain. Trends Neurosci. 13, 335-339.

(Accepted 10 March 1994) 